\title{
Effect of Tetramethylammonium Hydroxide on Nucleation, Surface Modification and Growth of Magnetic Nanoparticles
}

\author{
Ângela L. Andrade, ${ }^{1,2}$ José D. Fabris, ${ }^{3}$ José D. Ardisson, ${ }^{4}$ Manuel A. Valente, ${ }^{5}$ \\ and José M. F. Ferreira ${ }^{1}$ \\ ${ }^{1}$ Department of Ceramics and Glass Engineering, CICECO, University of Aveiro, 3810193 Aveiro, Portugal \\ ${ }^{2}$ Department of Chemistry, ICEB, Federal University of Ouro Preto, 35400-000 Ouro Preto, MG, Brazil \\ ${ }^{3}$ Federal University of Jequitinhonha and Mucuri Valleys (UFVJM), 39100-000 Diamantina, MG, Brazil \\ ${ }^{4}$ Applied Physics Laboratory, Nuclear Technology Development Center, (CDTN)/CNEN, 31270-901 Belo Horizonte, MG, Brazil \\ ${ }^{5}$ Department of Physics, University of Aveiro, I3N, 3810193 Aveiro, Portugal
}

Correspondence should be addressed to José M. F. Ferreira, jmf@ua.pt

Received 26 March 2012; Accepted 11 June 2012

Academic Editor: Leonard Deepak Francis

Copyright (๑) 2012 Ângela L. Andrade et al. This is an open access article distributed under the Creative Commons Attribution License, which permits unrestricted use, distribution, and reproduction in any medium, provided the original work is properly cited.

\begin{abstract}
Nanoparticles of magnetite $\left(\mathrm{Fe}_{3} \mathrm{O}_{4}\right)$ were obtained by reacting ferric chloride with sodium sulphite, through the reductionprecipitation method. The effects of adding tetramethylammonium hydroxide (TMAOH) during or after the precipitation of the iron oxide were studied in an attempt to obtain well-dispersed magnetite nanoparticles. Accordingly, the following experimental conditions were tested: (i) precipitation in absence of TMAOH (sample Mt), (ii) the same as (i) after peptizing with TMAOH (Mt1), (iii) TMAOH added to the reaction mixture during the precipitation of magnetite (Mt2). Analyses with transmission electron microscopy (TEM), X-ray diffraction, Mössbauer spectroscopy, attenuated total reflectance Fourier transform infrared spectroscopy (ATR-FTIR), zeta potential, and magnetization measurements up to $2.5 \mathrm{~T}$ revealed that magnetite was normally formed also in the medium containing TMAOH. The degree of particles agglomeration was monitored with laser diffraction and technique and inspection of TEM images. The relative contributions of Néel and Brownian relaxations on the magnetic heat dissipation were studied by investigating the ability of suspensions of these magnetite nanoparticles to release heat in aqueous and in hydrogel media. Based on ATR-FTIR and zeta potential data, it is suggested that the surfaces of the synthesized magnetite particles treated with TMAOH become coated with $\left(\mathrm{CH}_{3}\right)_{4} \mathrm{~N}^{+}$cations.
\end{abstract}

\section{Introduction}

Studying fundamental properties of synthetic analogous magnetite $\left(\mathrm{Fe}_{3} \mathrm{O}_{4}\right)$ nanoparticles keeps drawing much scientific attention and technological interest mainly due to their actual and many potential uses on data storage $[1,2]$, biolabeling, and on the separation of biomolecules $[3,4]$. Most of those applications require chemically stable and uniformly sized nanoparticles that will be well dispersed in liquid media. Usually, a protection layer on the particle surface is necessary to ensure their chemical stability and improve their dispersion ability, in order to increase the surface area to volume ratios. $\mathrm{Fe}_{3} \mathrm{O}_{4}$ nanoparticles are inherently unstable and tend to spontaneously aggregate to minimize their high surface energies.
Stable concentrated suspensions of magnetic nanoparticles in either organic or inorganic solvents are known as magnetic fluids or ferrofluids [5]. Magnetic fluids were firstly synthesized in 1964, by Papell [6]. They behave as a functional fluid and have increasingly found technological applications in a variety of fields, such as electronic packing, mechanical engineering, aerospace, and bioengineering [7]. Over the recent years, new ferrofluids and their derivatives have been developed for medical diagnosis and therapeutic practices in oncology, specifically, in these cases, for materials with magnetic ferrofluid hyperthermia (MFH) $[8,9]$, and for many other biomedical technologies [10-13].

Hyperthermia is a physical phenomenon that fundaments a medical practice in cancer therapy. The practice consists in selectively heating the malignant tumor, by 
placing the magnetocaloric material closely to or in direct contact with the affected internal organ of human body. Those organs tend to have fewer blood vessels and are less oxygenated than health ones. Consequently, they are more sensible and die when the local temperature goes above $43^{\circ} \mathrm{C}$.

The heat dissipation from magnetic particles is caused by the delay of the relaxation of the magnetic moment through either the rotation within the particle (Néel) or the rotation of the particle itself (Brownian), when they are exposed to an AC-magnetic field with magnetic field reversal times shorter than the magnetic relaxation times of the particles [14]. In 2009, Suto et al. [14] showed that the relative contribution between Néel and Brownian relaxations varies depending upon the particle size. Differently from the Brownian relaxation, the heat dissipated through Néel relaxation is not influenced by viscosity of the medium. For example, if the viscosity of the medium is high or if the rotational degree of freedom of the particle restricted, the heat dissipated may either diminish or even completely seize. It is always necessary to determine the relative simultaneous contribution of heat from Néel and Brownian relaxations, to estimate the possible minimum and maximum heat that could be generated with in vivo experiments.

Berger et al. [15] have successfully prepared a ferrofluid of $\mathrm{Fe}_{3} \mathrm{O}_{4}$ by reacting iron (II) and iron (III) ions in an aqueous ammonia solution with the addition of tetramethylammonium hydroxide (TMAOH), in order to chemically stabilize the magnetic nanoparticles in a colloidal suspension. Cheng et al. [16] have synthesized ferrofluids of magnetite by coprecipitating iron (II) and iron (III) ions in an aqueous solution alkalinized with TMAOH. According to Jolivet et al. [17], TMAOH acts as a surfactant to nanoparticles by absorbing the cationic species at the surface $\mathrm{OH}$ groups thus creating an electrostatic repulsion layer surrounding the particles keeping them apart. On the other hand, a number of earlier studies by Yang et al. [1823] on hydrothermal synthesis of $\mathrm{TiO}_{2}[18-22]$ and $\alpha-\mathrm{Al}_{2} \mathrm{O}_{3}$ nanoparticles [23] confirmed that TMAOH plays a very active peptizing role, enhancing the state of dispersion and the crystallinity of the synthesized materials.

In the present work, $\mathrm{Fe}_{3} \mathrm{O}_{4}$ nanoparticles were synthetized via precipitation of partially reduced ferric chloride with sodium sulfide, in an alkaline solution. The effects of adding TMAOH either during the precipitation or after the synthesis of magnetite on the characteristics of the nanoparticles and on the colloidal stability of the suspensions will be discussed in this report.

\section{Experimental Procedure}

2.1. Reagents. All chemicals used, such as iron (III) chloride hexahydrate, $\mathrm{FeCl}_{3} \cdot 6 \mathrm{H}_{2} \mathrm{O}$ (Riedel-de Haen, France); sodium sulfite, $\mathrm{Na}_{2} \mathrm{SO}_{3}$ (Sigma-Aldrich, Japan); ammonium hydroxide, $\mathrm{NH}_{4} \mathrm{OH}$ (Fluka, Germany); $25 \%$ aqueous tetramethylammonium hydroxide solution, $\mathrm{C}_{4} \mathrm{H}_{13} \mathrm{NO} \cdot 5 \mathrm{H}_{2} \mathrm{O}(\mathrm{TMAOH})$ (Aldrich, Germany, Japan) and hydrochloric acid, $\mathrm{HCl}$ (Sigma-Aldrich) were of analytical grade and used as received.
2.2. Synthesis of Iron Oxide Nanoparticles. Samples of $\mathrm{Fe}_{3} \mathrm{O}_{4}$ nanoparticles were obtained via the reduction-precipitation method, following the procedure described in details elsewhere [24]. In one of them, the iron ions solution was alkalinized with ammonium hydroxide and the particles were peptized with TMAOH. In the other, the iron ions solution was directly alkalinized with the peptizing agent (TMAOH), as described below.

2.2.1. Alkalinizing with Ammonia. $30 \mathrm{~mL}$ of a $\mathrm{FeCl}_{3} \cdot 6 \mathrm{H}_{2} \mathrm{O}$ stock solution containing $0.5 \mathrm{~mol} \mathrm{~L}^{-1}$ (dissolved in $\left.0.5 \mathrm{~mol} \mathrm{~L}^{-1} \mathrm{HCl}\right), 20 \mathrm{~mL}$ of a $\mathrm{Na}_{2} \mathrm{SO}_{3}$ stock solution with $1 \mathrm{~mol} \mathrm{~L}^{-1}$, and $50.8 \mathrm{~mL}$ of a $\mathrm{NH}_{4} \mathrm{OH}$ solution diluted to a total volume of $800 \mathrm{~mL}$ were used. Just after mixing $\mathrm{Fe}^{3+}$ and $\mathrm{SO}_{3}{ }^{-2}$, the color of the solution changed from yellow to red and, after few minutes, the yellow color reappeared. A diluted ammonia solution was then quickly poured into the mixture under vigorous stirring; a black precipitate was then formed. Stirring continued for an additional $30 \mathrm{~min}$. The $\mathrm{pH}$ of the solution was monitored to be maintained at 10.0 \pm 0.1 . The suspension containing the precipitated powder was centrifuged at 2,000 rpm for $3 \mathrm{~min}$; the supernatant was discarded. This procedure was repeated for five times by redispersing the resultant cakes in distilled water, each time. The as-obtained precipitate sample was labeled Mt. The Mt nanoparticles were peptized with TMAOH. Typically, $1 \mathrm{~mL}$ of 25 mass $\%$ TMAOH solution was added to each centrifuge tube containing an amount of wet cake corresponding to about $1 \mathrm{~g}$ of dry powder and re-dispersed by stirring with a thin glass rod until obtaining homogeneous suspensions. These suspensions were then dried to obtain the powders for the different measurements. This sample peptized with TMAOH was labeled Mt1.

2.2.2. Alkalinizing with TMAOH. In this case, magnetite nanoparticles were synthesized in the same way as described above, but alkalinizing was made by quickly pouring 25 mass $\%$ TMAOH solution until a pH of about $10.0 \pm 0.1$ was obtained. A precipitate was formed. The mixture was stirred for further $30 \mathrm{~min}$. The obtained suspension was centrifuged at 2,000 rpm for $3 \mathrm{~min}$, and the supernatant was discarded. This procedure was repeated five times by redispersing the resultant cakes in distilled water. The centrifuged cakes were dried to obtain the powders to be used for further characterization. The obtained solid sample, precipitated in medium containing TMAOH, was labeled Mt2.

2.3. Characterization Techniques. For transmission electron microscopy analysis (TEM), a drop of the suspension of the magnetite sample was placed onto a copper mesh coated with an amorphous carbon film and then dried in an evacuated desiccator. TEM micrographs were recorded on a transmission electron microscope (Hitachi, 9000 NA, Japan) and the particle size was measured with the software Image J. The crystalline phases were determined by X-ray powder diffraction (XRD) analysis (Rigaku Geigerflex D/Max, C Series; $\mathrm{CuK} \alpha$ radiation; $2 \theta$ angle range $20^{\circ}-70^{\circ}$; step $0.02 \mathrm{~s}^{-1}$ ) after evaporation of the liquid carrier. Phases were 
identified by comparing the experimental X-ray patterns with standard files compiled by the International Centre for Diffraction Data. The Rietveld structural refinement was performed with FULLPROF 2010 program. Mössbauer spectra were collected in constant acceleration transmission mode with a $\sim 50 \mathrm{mCi}{ }^{57} \mathrm{Co} / \mathrm{Rh}$ gamma-ray source. Spectra at $298 \mathrm{~K}$ and $100 \mathrm{~K}$ were obtained with a spectrometer equipped with a transducer (CMTE model MA250) controlled by a linear function driving unit (CMTE model MR351). Values of Mössbauer isomer shifts are quoted relatively to $\alpha$-Fe. The experimental reflections were fitted to Lorentzian functions by least-square fitting with software NORMOS-90 (this NORMOS package was developed by R. A. Brand, Laboratorium für Angewandte Physik, Universität Duisburg, D-47048, Duisburg-Germany). The ATR-FTIR data were collected with a FT-IR model Mattson Galaxy S-7000, 32 scans, and resolution of $4 \mathrm{~cm}^{-1}$.

The determination of zeta potential $(\xi)$ was performed on a COULTER DELSA 440SX, by using a stock suspension of the ground material in deionized water, prepared and homogenized by ultrasonication for $15 \mathrm{~min}$. Drops of this stock suspension were then added to an aqueous solution of $\mathrm{KCl} 10^{-3} \mathrm{~mol} \mathrm{~L}^{-1}$ for the zeta potential measurements. The $\mathrm{pH}$ of the measuring solution was varied and adjusted to several values, in the $\mathrm{pH}$ range of 3.5 to 10.5 , by using $10^{-3} \mathrm{~mol} \mathrm{~L}-1$ aqueous solutions of $\mathrm{KOH}$ and $\mathrm{HNO}_{3}$.

The agglomerate grain sizes in suspension were determined by dynamic light scattering (DSL) using a Zetasizer Nano ZS instrument (Malvern Instruments Ltd., UK) that allows specific measurements of the $z$ average diameter (defined as the intensity-weighted average hydrodynamic diameter of the particles being measured). An aliquot of a stock suspension of the ground material in deionized water was prepared and homogenized by ultrasonication for $5 \mathrm{~min}$. The used water was purified by passing through a $0.22 \mu \mathrm{m}$ Millipore filter. The $z$-average diameter was calculated as the average of six measurements.

The DC magnetic measurements were performed on powder samples (100-150 mg) using a vibrating sample magnetometer (VSM) with a cryogen-free magnet (cryogeniccryofree), at the University of Aveiro, Portugal. Typical hysteresis curves were obtained at $300 \pm 0.1 \mathrm{~K}$ with a magnetic field varying between -2.5 and $2.5 \mathrm{~T}$. The magnetic parameters such as saturation magnetization $\left(\mathrm{M}_{\mathrm{s}}\right)$, coercivity $\left(\mathrm{H}_{\mathrm{c}}\right)$, and retentivity $\left(\mathrm{M}_{\mathrm{r}}\right)$ were obtained from the VSM results.

Heat dissipation experiments were carried out by transfering the suspensions with dispersed magnetite in both water and hydrogel into a test tube. This tube was placed at the center of a three-loop coil of the VSM equipment, consisting of a power supply (Nova Star 5 kW RF Power Supply, Ameritherm, Inc, Scottsville, NY, USA) and a heating station (Induction atmospheres). The sample concentration was approximately $2 \mathrm{mg} \mathrm{mL}^{-1}$ in water and $2 \mathrm{mg} \mathrm{g}^{-1}$ in polyvinyl alcohol hydrogel. In the hydrogel dispersed samples, rotation of particles was restricted and the magnetic moment relaxed only through Néel relaxation. The temperature of the magnetic suspension was measured with an optical fiber thermometer. Results were taken as the mean of triplicate measurements.
The polyvinyl alcohol hydrogel was based on the method earlier proposed by Hyon et al. [25], with minor modifications. A homogeneous polyvinyl alcohol (PVA) solution with a PVA concentration of $15 \mathrm{wt} \%$ was obtained by heating the mixture of PVA and a mixed water/dimethyl sulfoxide (DMSO) solvent at $140^{\circ} \mathrm{C}$ for $2 \mathrm{hrs}$. The mixing ratio of water to DMSO was kept to $20 / 80$ by weight. Then the casted PVA was placed in a freezer at $-20^{\circ} \mathrm{C}$ for $24 \mathrm{~h}$.

\section{Results and Discussion}

Centrifuging the just formed precipitate, still in aqueous suspension, in order to finally obtain the Mt2 sample, at $2,000 \mathrm{rpm}$ for $3 \mathrm{~min}$ was found to be enough condition to separate the solid particles from the liquid phase, whereas for the corresponding Mt1 sample, centrifugation even at $12,000 \mathrm{rpm}$ for $6 \mathrm{~min}$ was insufficient to separate the two phases. This suggests that Mt2 particles are coarser and/or strongly agglomerated than Mt1.

The obtained Mt, Mt1, and Mt2 samples were black in color and exhibited magnetic behavior, as it could be observed from their response to the magnetic field of a small hand magnet. The Mtl suspension kept their colloidal characteristics for up to 5 months with negligible sedimentation in this ferrofluid. Contrarily, the Mt2 sample was completely sedimented when the ferrofluid flask was in rest for $1 \mathrm{~h}$, evidencing again the coarser and/or aggregated nature of its particles.

The $z$-average diameter of the Mt, Mt1, and Mt2 samples in water was as follows: $243.7 \pm 18.59,85.86 \pm 2.736$, and $1,906 \pm 495.8 \mathrm{~nm}$, respectively. The large individual or aggregated grains, as observed for sample Mt2, explains the reason by which it sedimented even faster than the Mt1 sample. The Mt1 ferrofluid is also much better dispersed than Mt and Mt2.

Figure 1 shows TEM images of Mt, Mt1, and Mt2 nanoparticles. The mean particle size diameters for the samples Mt, Mt1, and Mt2 were found to be about $5.7 \pm$ $2.9 \mathrm{~nm}, 5.8 \pm 2.7 \mathrm{~nm}$, and $9.7 \pm 6.4 \mathrm{~nm}$, respectively. The mean size of the particles/aggregates is larger in the case of Mt2 sample. The higher tendency of particles in sample Mt2 to be aggregated can be explained by the bigger particle sizes but also by their higher crystallinity (Figure 2), well favored by the addition of the peptizing agent (TMAOH). Larger particles of well-crystallized magnetite in which the bulk properties supersede surface properties, and they are expected to magnetically attract more strongly each other. Hosono et al. [26] suggested that the bigger particles could be a consequence of higher concentration of charged ions in the solution since agglomeration between particles occurs by shrinkage of electric double layer. Moreover, the role of the ionic strength on the particle size also is largely dependent on the nature of the electrolyte. The smallest cations being the best screening ions, their influence on the surface charge is highest. The surface of iron oxides is negatively charged at $\mathrm{pH}>\mathrm{PZC}$ (point of zero charge). These characteristics explain the strong influence of $\mathrm{NH}_{4}{ }^{+}$on reducing particle sizes, as compared to the $\left[\mathrm{N}\left(\mathrm{CH}_{3}\right)_{4}\right]^{+}$cations, and also on 

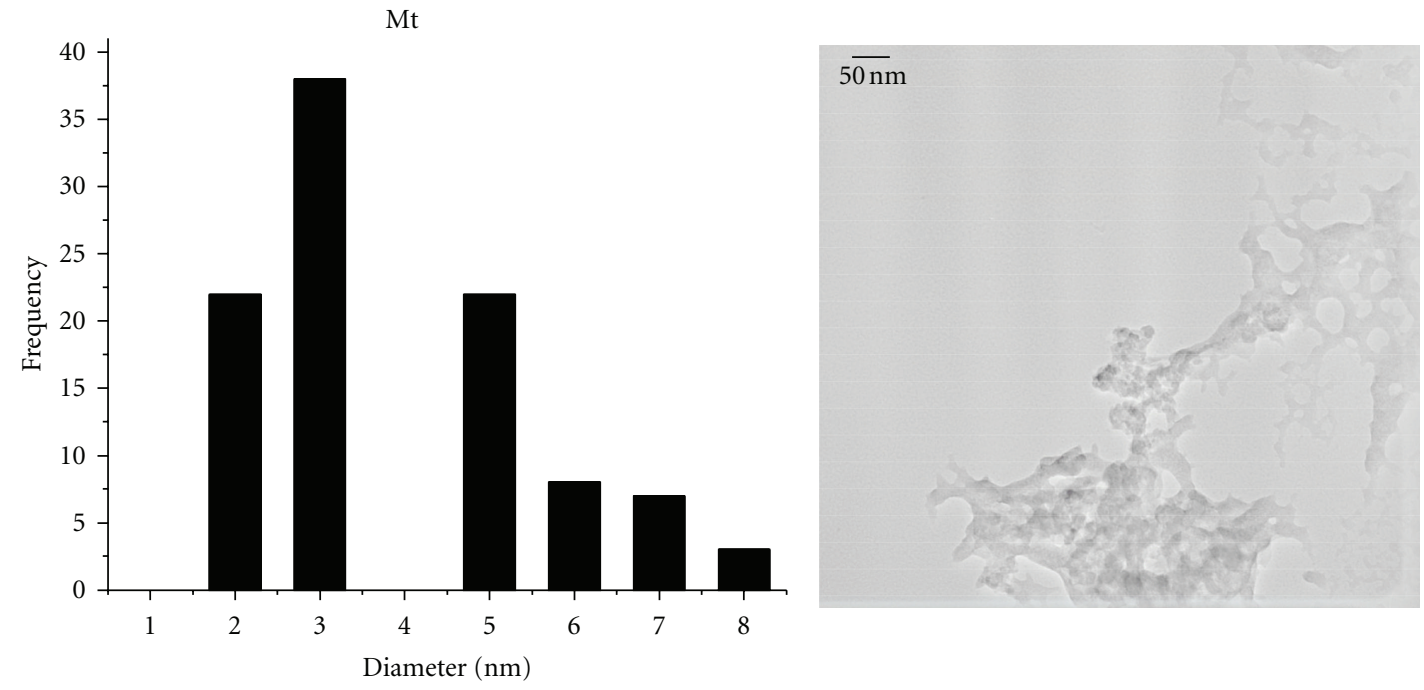

(a)
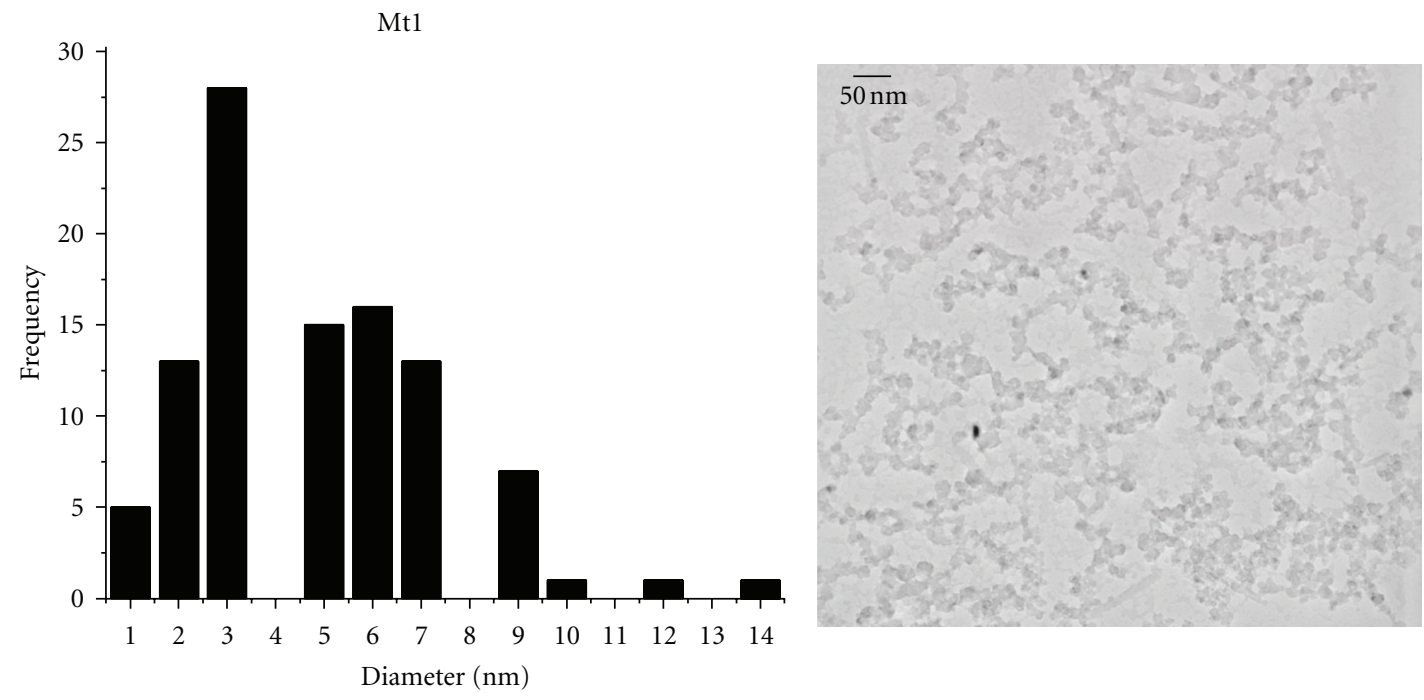

(b)
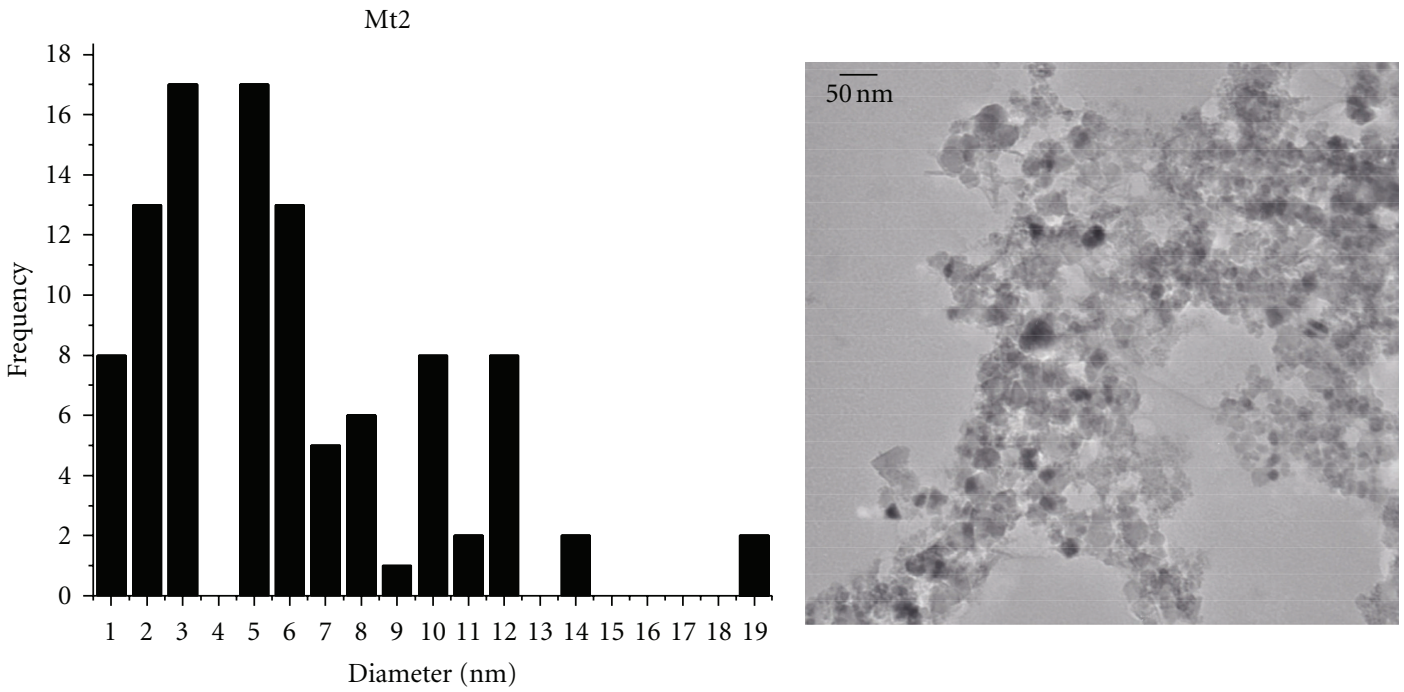

(c)

FIGURE 1: TEM micrograph of samples: Mt, Mt1, and Mt2. 


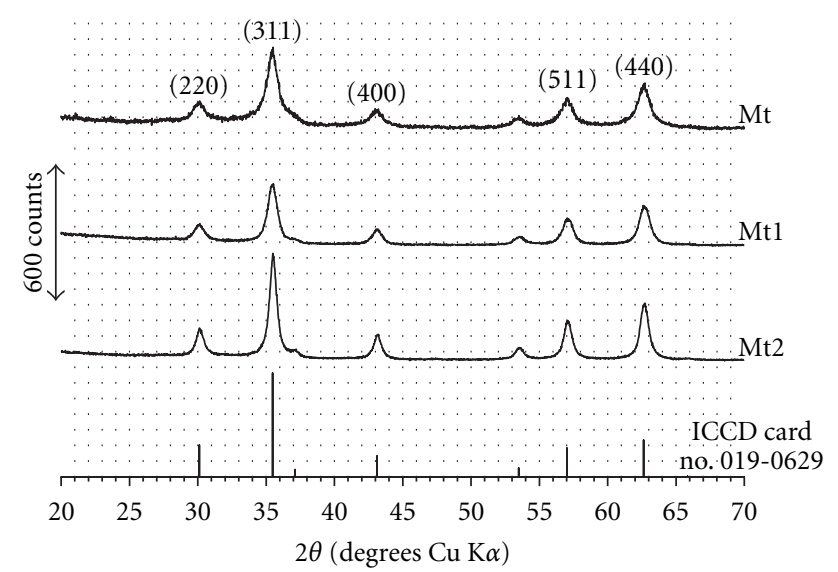

Figure 2: X-ray diffraction patterns of samples: Mt, Mt1, Mt2, and magnetite (ICDD file no. 019-0629).

preventing particles aggregation. This interpretation is in line with that of Bacri et al. [27, 28], who proposed a decreasing stability of the iron oxide nanoparticles as a consequence of a decrease of the electrical double layer thickness around the particles, driven by the increased ionic strength of the media upon adding TMAOH.

The crystalline structure of the synthesized iron oxide species analyzed by powder XRD (Figure 2) shows that $\mathrm{Fe}_{3} \mathrm{O}_{4}$ was identified in these Mt, Mt1, and Mt2 samples. The most intense reflections for this phase were registered at $2 \theta=$ $30.095^{\circ}, 35.423^{\circ}, 43.053^{\circ}, 57.22^{\circ}$ and $62.76^{\circ}$, corresponding to the planes of (220), (311), (400), (511), and (440), respectively, of the iron oxide spinel. The peaks in the diffractograms match well with the PDF data for magnetite from the powder diffraction file (PDF) from International Centre for Diffraction Data (ICDD) card number 019-0629. The average particle sizes for $\mathrm{Mt}, \mathrm{Mt} 1$, and $\mathrm{Mt} 2$ magnetites, from breadths of reflection 311, estimated with Scherrer equation were 11, 10, and $13 \mathrm{~nm}$, respectively [29]. From routine powder XRD data only, it may be difficult to differentiate the coexisting maghemite $\left(\gamma \mathrm{Fe}_{2} \mathrm{O}_{3}\right)$ and magnetite $\left(\mathrm{Fe}_{3} \mathrm{O}_{4}\right)$ in a same sample since structure is cubic with relatively close unit cell dimension. The diffraction patterns for both phases are quite similar. Therefore, one might presume that magnetite and maghemite are components of the obtained ferromagnetic phases, as oxidation processes could not be totally avoided during chemical synthesis and solid powder washings and handling in air. Results in Figure 2 confirm that the sample Mt that has not been in contact with the peptizing agent exhibits the lowest degree of crystallinity as it can be drawn from the noisy background and from the broader diffraction peaks. Contrarily, the Mt2 sample that has been synthesized from the very beginning in presence of TMAOH presents the sharpest XRD diffraction peaks, which is in good consistence with its coarser morphology (Figure 1). When the peptizing agent was added to the precipitated Mt sample, it promoted a better dispersion of the partially amorphous nanoparticles and enhanced the degree of spatial arrangement of the matter, thus increasing the crystallinity. This effect was reportedly proposed for other systems [18-23]. These results suggest that, in the case

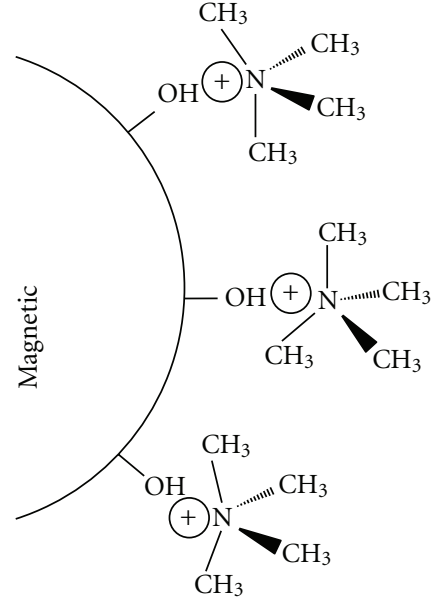

Figure 3: Peptization exerted by tetramethyl ammonium cations adsorbed onto the surface of magnetic nanoparticles thus, creating repulsive forces and enabling the particle growing species to be added to the surface in an organized manner.

of $\mathrm{Mt} 2$, the $\left[\left(\mathrm{CH}_{3}\right)_{4} \mathrm{~N}\right]^{+}$cations are prone to readily adsorb specifically onto the surface of the early nuclei being formed, as schematized in Figure 3, hindering some of them to achieve the critical size for growing. Under this perspective, a smaller number of effective nuclei would be formed, explaining the greater size of the resulting nanoparticles in the case of Mt2 sample.

Mössbauer spectra obtained at variable temperatures (Figure 4) reveal that these nanosized particles do contain ferric iron oxides in small particle sizes, more clearly for samples Mt and Mt2.

These magnetic nanoparticles in a liquid medium experience reorientations of the magnetization vector, in single anisotropic magnetic domains (Néel relaxation time, $\tau_{\mathrm{N}}$ ) but also due to viscous rotation of the particle (Brownian relaxation time, $\left.\tau_{\mathrm{B}}\right)[30,31]$.

The global magnetic relaxation times $(\tau)$ of colloids are the sum of the Néel and Brownian relaxation rates, which is given by $[31,32]$

$$
\frac{1}{\tau}=\frac{1}{\tau_{\mathrm{B}}}+\frac{1}{\tau_{\mathrm{N}}}
$$

These relaxations explain the obtained Mössbauer spectra with a relatively intense central $\mathrm{Fe}^{3+}$ doublet along with a magnetic sextet, as it was more clearly observed for samples $\mathrm{Mt}$ and Mt2. However, lowering the sample temperature tends to progressively block the relaxations effect and promote the magnetic ordering. The coexistence of these two major spectral contributions, that is, superparamagnetic and magnetically ordered patterns, in different proportions, means that particle sizes distributions are different in each case. From Mössbauer data only, the sample Mt does contain higher proportion of smaller particles than in Mt2, but sample Mt1 contains the highest proportion of bigger particles of all three. The corresponding hyperfine field distributions clearly reflect these trends. The $80 \mathrm{~K}$ probability profile for sample $\mathrm{Mt1}$ is considerably narrower 


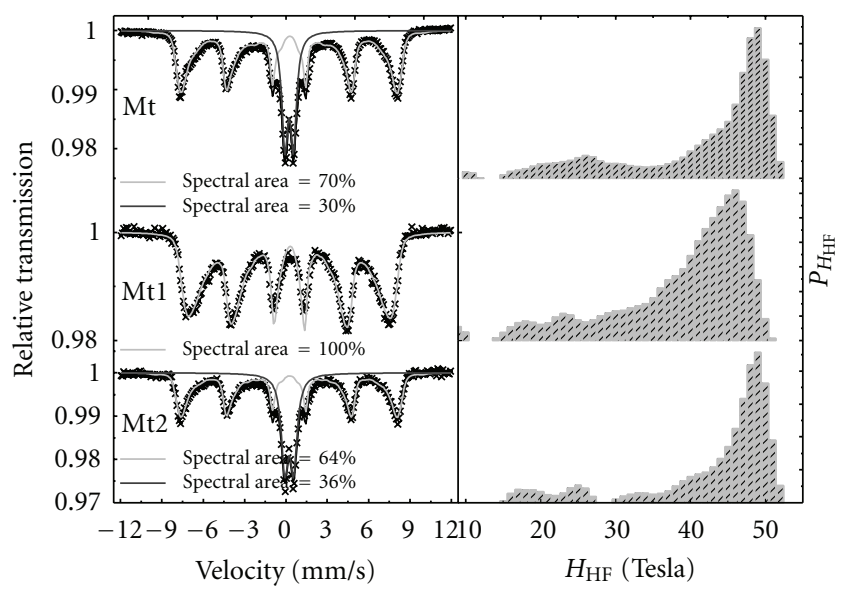

(a)

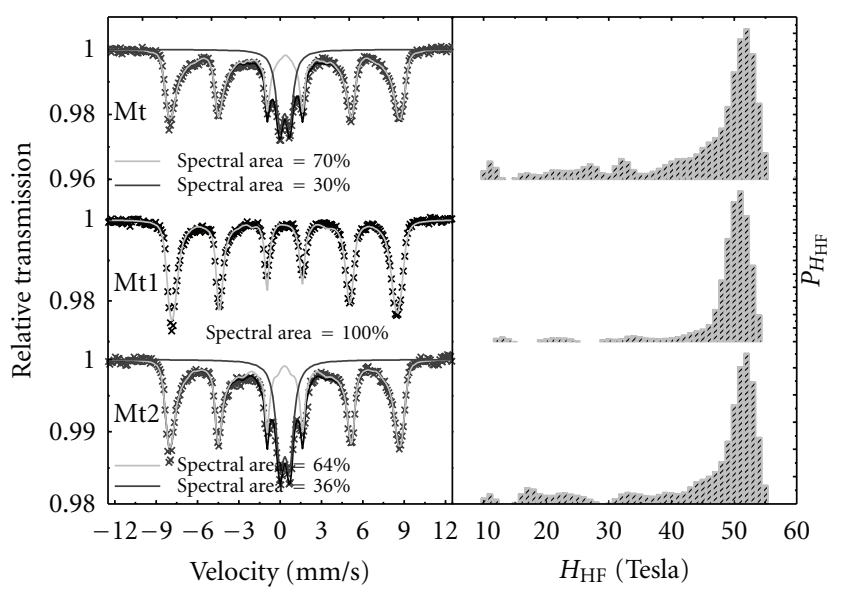

(b)

Figure 4: Mössbauer spectra of Mt, Mt1, and Mt2 at (a) RT, and ( b) $80 \mathrm{~K}$.

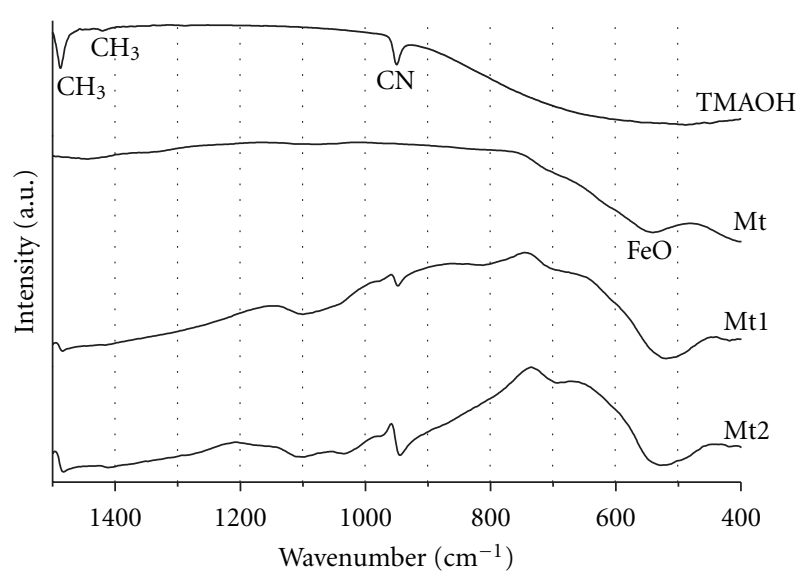

FIgURE 5: ATR-FTIR spectra of TMAOH, Mt, Mt1, and Mt2.

than for the other two samples. Values of hyperfine magnetic fields at maximum probability for all these magnetic samples steadily increase on going from $297 \mathrm{~K}$ to $80 \mathrm{~K}$, following values well around those expected for maghemite $\left(\gamma \mathrm{Fe}_{2} \mathrm{O}_{3}\right)$.

The apparent contradiction with evidences from hyperthermic measurements that point to sample Mt2 as the sample containing bigger particles than $\mathrm{Mt}$ and $\mathrm{Mt} 1$ is explained by assuming that the Brownian relaxation rate in sample Mt2, with more strongly aggregated nanoparticles, is much slower so to produce a more significant hyperthermic effect. However, Mössbauer spectra are necessarily collected with the solid sample. In this condition, the global relaxation time is only influenced by the Néel relaxation, for which magnitude, particularly for samples $\mathrm{Mt}$ and $\mathrm{Mt} 2$, would be comparable or below the time-scale window for Mössbauer spectroscopy, or $\sim 10^{-7} \mathrm{~s}$.

A Mössbauer spectrum results from the hyperfine coupling arising from extranuclear electric and magnetic fields that influence the energy levels of the probe nucleus. The fast fluctuation of the magnetization vector between easy directions in the monodomain nanosystem, relatively to the lifetime decay of the ${ }^{57} \mathrm{Fe} 14.4 \mathrm{keV}$ Mössbauer level, would lead to an electric quadrupole coupling, resulting in a spectral superparamagnetic doublet. Particles aggregation that is promoted by coating their surface with surfactant or polymerizing organic materials may be enough to change the hyperthermic behavior of the material but not necessarily the hyperfine structure of the solid material. Aggregates of fine particles may present typical hyperthermic behavior as of bigger particles, essentially due to the increasing Brownian reorientation energy in the colloidal medium, but their hyperfine structure still accounts for local interactions at the atomic nuclear level in the superparamagnetic state of the solid material.

Figure 5 compares the ATR-FTIR spectra of the TMAOH and of the Mt, Mt1, and Mt2 samples. The ATR-FTIR spectrum of the TMAOH shows a strong band at $1490 \mathrm{~cm}^{-1}$, assigned to the asymmetric methyl deformation mode, $\delta_{\text {asym }}\left(\mathrm{CH}_{3}\right)$. A smaller one assigned to the symmetrical methyl deformation mode $\delta_{\text {sym }}\left(\mathrm{CH}_{3}\right)$ appears near to $1430 \mathrm{~cm}^{-1}$. A single band appeared at $950 \mathrm{~cm}^{-1}$ is attributed to the $v_{\text {asym }}(\mathrm{C}-\mathrm{N})$ mode which is generally observed in the domain $900-1000 \mathrm{~cm}^{-1}$ [33]. The Mt sample shows a band at $550 \mathrm{~cm}^{-1}$. Since magnetite has an inverse spinel-type structure, it shows bands indicating the vibrations $\mathrm{M}_{\mathrm{T}}-\mathrm{O}-$ $\mathrm{M}_{O}\left(\nu 1 \approx 600-550 \mathrm{~cm}^{-1}\right)$, where $\mathrm{M}_{\mathrm{T}}$ and $\mathrm{M}_{\mathrm{O}}$ correspond to the metal occupying tetrahedral and octahedral positions, respectively [34-36]. The ATR-FTIR spectra of the Mt1 and Mt2 samples also show the band at $550 \mathrm{~cm}^{-1}$ characteristic of magnetite and the characteristic vibration of $\mathrm{Fe}-\mathrm{O}$ in maghemite $\left(\gamma-\mathrm{Fe}_{2} \mathrm{O}_{3}\right)$ at $690 \mathrm{~cm}^{-1}[37-39]$, as well as the transmittance bands typical of $\mathrm{TMAOH}$, proving that the peptizing agent has been strongly adsorbed at the surface of the nanoparticles.

Zeta potential data are a powerful tool being extensively used to evaluate the effects of the presence of surfaceactive agents in different systems. In the present work, this technique was also used to help evaluating the influence of 


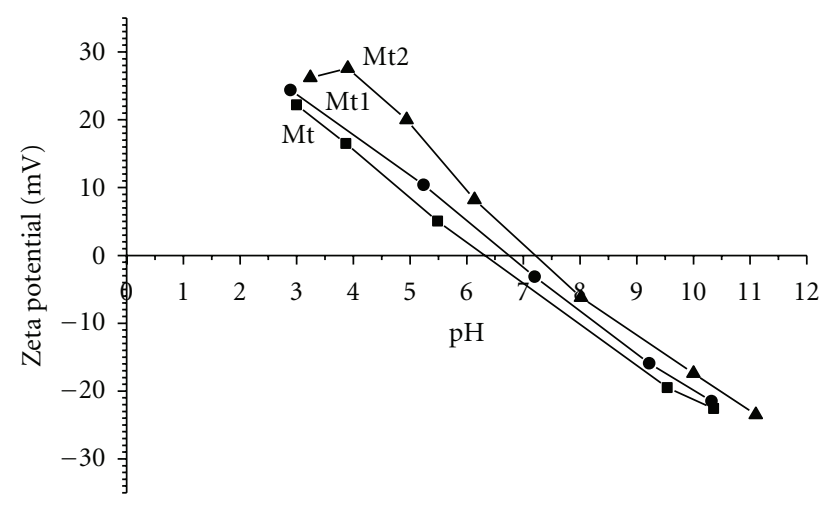

Figure 6: Variation of zeta potential of samples: Mt, Mt1, and Mt2.

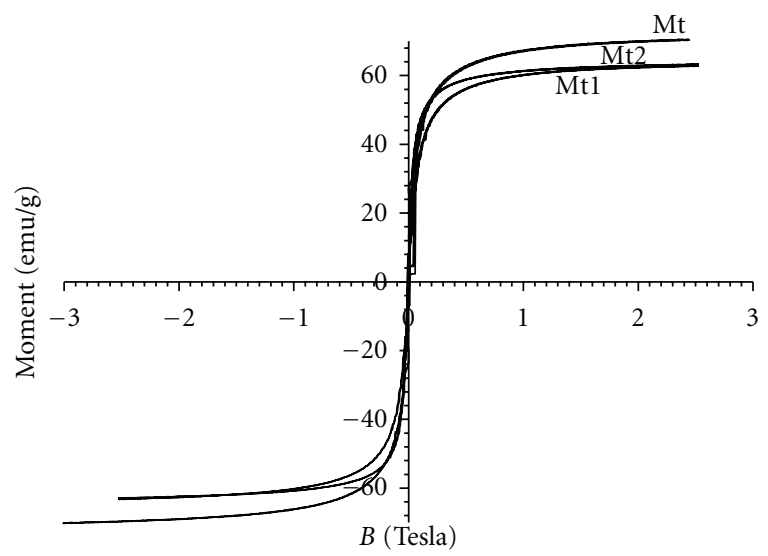

Figure 7: Magnetization curves of samples: Mt, Mt1, and Mt2.

added TMAOH on the solid/liquid interface properties of magnetite nanoparticles. Results presented in Figure 6 show that the $\mathrm{pH}$ values corresponding to the isoelectric points $\left(\mathrm{pH}_{\mathrm{IEP}}\right)$ of the Mt and Mt1 samples were found to be about 6.4 and 6.8, respectively. This is in good agreement with the reported data in the scientific literature for magnetite $[40,41]$. In the Mt2 sample, the $\mathrm{pH}_{\mathrm{IEP}}$ was about 7.3. The increase in $\mathrm{pH}_{\text {IEP }}$ of the Mt1 and Mt2 samples, with respect to that determined for $\mathrm{Mt}$ sample, indicates that $\left[\left(\mathrm{CH}_{3}\right)_{4} \mathrm{~N}\right]^{+}$ cations were specifically adsorbed onto the surface of the synthesized iron oxide species [42]. This specific adsorption is partially driven by the negative charge exhibited by magnetite above its $\mathrm{pH}_{\text {IEP }}$ (6.4) and has been apparently more extensive when the peptizing agent was added during the precipitation. Thus, the adsorbed $\left[\left(\mathrm{CH}_{3}\right)_{4} \mathrm{~N}\right]^{+}$ions tend to decrease the negative charge of the iron oxide powder at alkaline $\mathrm{pH}$ range.

Magnetization curves obtained for Mt, Mt1, and Mt2 samples at $300 \mathrm{~K}$ are presented in Figure 7. The curves indicate a superparamagnetic behavior, for all these samples, as evidenced by zero coercivity and remanence on the magnetization loop. The saturation magnetization $\left(M_{S}\right)$ was obtained from moment versus magnetic field $(B)$ curves. For the Mt sample, the saturation magnetization value is $70.7 \mathrm{emug}^{-1}$, being higher than values reported in the literature $\left(46.3 \mathrm{emu} \mathrm{g}^{-1}\right)$ for samples prepared by the
TABLE 1: The $\Delta T_{\max }$ values of the magnetite samples dispersed in water and hydrogel under 220 Oe AC magnetic field.

\begin{tabular}{lcc}
\hline Sample & \multicolumn{2}{c}{$\Delta T_{\max }$} \\
\hline Mt & Water & Hydrogel \\
Mt1 & 23 & 12 \\
Mt2 & 11 & 6 \\
\hline
\end{tabular}

same method [43], whereas for peptized samples Mt1 and Mt2 with TMAOH the corresponding values are 62.8 and $63.2 \mathrm{emu} \mathrm{g}^{-1}$. The magnetic saturation values of magnetite nanoparticles are experimentally determined to be in the range of 30-50 emu g ${ }^{-1}$, which is lower than the bulk value, $90 \mathrm{emu} \mathrm{g}^{-1}$ [44]. Similar values have been obtained for Mt sample. It was expected that the saturation magnetization was higher for samples Mt1 and Mt2, as they have a higher degree of crystallinity and, in the case of Mt2, a larger particle size. The differences between the saturation magnetization between the samples with and without TMAOH can be ascribed to the previous presumption that magnetite and maghemite are both present in these peptized powders with TMAOH.

The magneto caloric behavior, influencing the heating ability of magnetite samples Mt, Mt- 1 , and Mt-2, is shown in Figure 8. For each experiment, the temperature rising was measured for every $1 \mathrm{~min}$ up to an accumulated time of $20 \mathrm{~min}$ and then every $5 \mathrm{~min}$ up to $1 \mathrm{~h}$. Superparamagnetic particles generate heat by conversion of magnetic energy into thermal energy caused by the delay in magnetic relaxation. According to the Néel model, the magnetic moment originally locked along the crystal easy axis rotates away from that axis, tending to align with the external field. The Néel mechanism is analogous to the hysteresis loss in multidomain magnetic particles whereby there is an internal friction due to the movement of the magnetic moment in an external field that results in heat generation. In the Brownian mode, the whole particle rotates in the direction of the field with the moment locked along the crystal axis. The heat generated through Néel or Brownian relaxation in an applied AC magnetic field depends on the size of the nanoparticles. Smaller nanoparticles generate heat preferentially by the Néel modell.

The amount of heat generated by pure water and hydrogel in 220 Oe AC magnetic fields was measured to obtain any contribution of water and hydrogel in the increase of temperature by the dispersed nanoparticles. The whole contribution of both pure water and gel was discounted from the final temperature value for each ferrofluid.

The results of heat dissipation experiments of the samples $\mathrm{Mt}, \mathrm{Mt} 1$, and $\mathrm{Mt} 2$ dispersed in water and hydrogel are shown in Figure 8. The $\Delta T_{\max }$ values for magnetite nanoparticles dispersed in water and hydrogel were determined from the gradients of temperature-time curves obtained by exposing the samples to an AC magnetic field strength of 220 Oe at frequency of $198 \mathrm{kHz}$. Results are summarized in Table 1. 


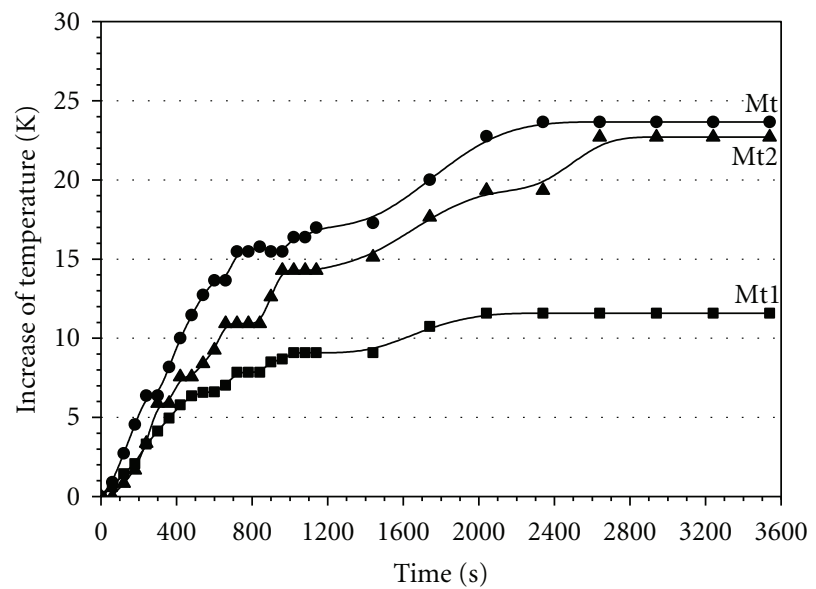

(a)

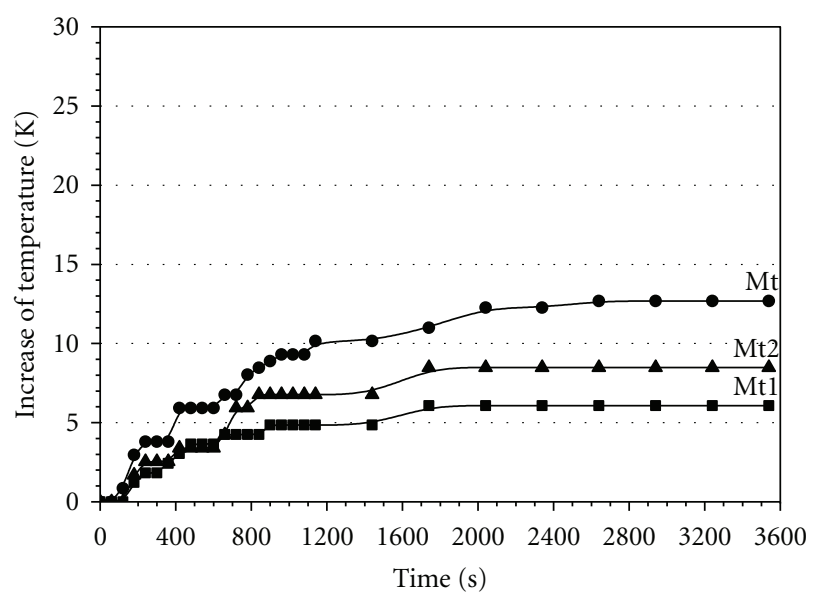

(b)

Figure 8: Temperature-time curves of the samples Mt, Mt1, and Mt2 dispersed in (a) water and (b) PVA hydrogel.

The $\Delta T_{\max }$ values for dispersed samples $\mathrm{Mt}, \mathrm{Mt1}$, and Mt2 in hydrogel were roughly 52, 54, and $42 \%$ less than that of the sole sample dispersed in water. The reduction in heat dissipation was attributed to the inhibition of particle rotation. In other words, particles should have an average Brownian magnetic relaxation time (TB) less than room temperature (RT) and narrow particle size distribution. This suggested that the fraction of the particles that generated heat through Brownian rotation was comparatively large. The sample with an average diameter below $13 \mathrm{~nm}$ had large fraction of particles dissipating heat through Néel relaxation losses. On the other hand, the sample with average diameter larger than $13 \mathrm{~nm}$ had large fraction of particles dissipating heat through Brownian relaxation [14]. Furthermore, these results suggested that it is important to analyze the relative contributions of Néel and Brownian relaxations losses to formulate the appropriate strategy for effective in vivo treatments. And also, it could be concluded that the samples with blocking temperature below RT are more suitable for a reliable in vivo MFH treatment. Thus, to derive at the appropriate sample for in vivo experiments, similar study on size-classified particles with TB less than RT and narrow size distribution should be pursued.

Hyperthermia is a cancer therapy that consists in heating selectively tumour in focusing organ zones. Those zones have fewer blood vessels and are less oxygenated than health ones. Consequently, they are more sensible and can die if the local temperature increases above $43^{\circ} \mathrm{C}$. Thus, a temperature variation of $\Delta T_{\max }=11^{\circ} \mathrm{C}$ would be sufficient to produce therapeutical effects.

\section{Conclusions}

Tetramethylammonium hydroxide (TMAOH) was revealed to be an effective peptizing agent for magnetite manoparticles in ferrofluid systems based on. The $\left[\left(\mathrm{CH}_{3}\right)_{4} \mathrm{~N}\right]^{+}$cations specifically adsorbed onto the surface of the synthesized iron oxide species give rise to repulsive stabilization forces that enable the particle growing species to be added to the surface in a more organized manner. Accordingly, when TMAOH is added during precipitation as in the case of Mt2 samples it promotes the formation of larger particles having a higher degree of crystallinity, including magnetite and maghemite. The addition of TMAOH after the magnetite has been synthesized promotes the colloidal stability of the nanoparticles and enhances the transformation of the amorphous material into more ordered crystalline phases.

\section{Acknowledgments}

This work was supported by $\mathrm{CNPq}$ (Brazil including Grants nos. 202212/2007-6 and 302479/2010-4), FAPEMIG (including Grantd no. PPM 00419-10 and no. APQ-00651-11) (Brazil) and by CICECO, (University of Aveiro, Portugal). CAPES (Brazil) grants the Visiting Professor PVNS fellowship to JDF at UFVJM.

\section{References}

[1] S. Sun, C. B. Murray, D. Weller, L. Folks, and A. Moser, "Monodisperse FePt nanoparticles and ferromagnetic FePt nanocrystal superlattices," Science, vol. 287, no. 5460, pp. 1989-1992, 2000.

[2] V. Skumryev, S. Stoyanov, Y. Zhang, G. Hadjipanayis, D. Givord, and J. Nogués, "Beating the superparamagnetic limit with exchange bias," Nature, vol. 423, no. 6942, pp. 850-853, 2003.

[3] M. Zhao, L. Josephson, Y. Tang, and R. Weissleder, "Magnetic sensors for protease assays," Angewandte Chemie, vol. 42, no. 12, pp. 1375-1378, 2003.

[4] P. S. Doyle, J. Bibette, A. Bancaud, and J. L. Viovy, "Selfassembled magnetic matrices for DNA separation chips," Science, vol. 295, no. 5563, p. 2237, 2002.

[5] R. E. Rosensweig, "Magnetic fluids," in Ferrohydrodynamics, Cambridge University Press, Cambridge, UK, 1985.

[6] S. S. Papell, US Patent No: 3215 572, 1965.

[7] Q. Li, Y. Xuan, and J. Wang, "Experimental investigations on transport properties of magnetic fluids," Experimental Thermal and Fluid Science, vol. 30, no. 2, pp. 109-116, 2005. 
[8] A. Jordan, R. Scholz, P. Wust et al., "Endocytosis of dextran and silan-coated magnetite nanoparticles and the effect of intracellular hyperthermia on human mammary carcinoma cells in vitro," Journal of Magnetism and Magnetic Materials, vol. 194, no. 1, pp. 185-196, 1999.

[9] D. C. F. Chan, D. B. Kirpotin, and J. P. A. Bunn, "Synthesis and evaluation of colloidal magnetic iron oxides for the site-specific radiofrequency-induced hyperthermia of cancer," Journal of Magnetism and Magnetic Materials, vol. 122, no. 13, pp. 374-378, 1993.

[10] C. Grob, K. Buscher, E. Romanus, C. A. Helm, and W. Weitschies, "Characterization of a ferrofluid by atomic force microscopy and photon correlation spectroscopy after magnetic fractionation," European Cells and Materials, vol. 3, supplement 2, pp. 163-166, 2002.

[11] L. M. Lacava, Z. G. M. Lacava, M. F. Da Silva et al., "Magnetic resonance of a dextran-coated magnetic fluid intravenously administered in mice," Biophysical Journal, vol. 80, no. 5, pp. 2483-2486, 2001.

[12] Z. G. M. Lacava, R. B. Azevedo, L. M. Lacava et al., "Toxic effects of ionic magnetic fluids in mice," Journal of Magnetism and Magnetic Materials, vol. 194, no. 1, pp. 90-95, 1999.

[13] M. Rǎcuciu, D. E. Creangǎ, V. Bădescu, and N. Sulitanu, "Microstructural investigation of some biocompatible ferrofluids," Journal of Magnetism and Magnetic Materials, vol. 316, no. 2, pp. e772-e775, 2007.

[14] M. Suto, Y. Hirota, H. Mamiya et al., "Heat dissipation mechanism of magnetite nanoparticles in magnetic fluid hyperthermia," Journal of Magnetism and Magnetic Materials, vol. 321, no. 10, pp. 1493-1496, 2009.

[15] P. Berger, N. B. Adelman, K. J. Beckman, D. J. Campbell, A. B. Ellis, and G. C. Lisensky, "Preparation and properties of an aqueous ferrofluid," Journal of Chemical Education, vol. 76, no. 7, pp. 943-948, 1999.

[16] F. Y. Cheng, C. H. Su, Y. S. Yang et al., "Characterization of aqueous dispersions of $\mathrm{Fe}_{3} \mathrm{O}_{4}$ nanoparticles and their biomedical applications," Biomaterials, vol. 26, no. 7, pp. 729738, 2005.

[17] J. P. Jolivet, R. Massart, and J. M. Fruchart, "Synthesis and physicochemical study of non-surfactant magnetic colloids in an aqueous-medium," Nouveau Journal De Chimie-New Journal of Chemistry, vol. 7, no. 5, pp. 325-331, 1983.

[18] J. Yang, S. Mei, and J. M. F. Ferreira, "Hydrothermal synthesis of nanosized titania powders: influence of peptization and peptizing agents on the crystalline phases and phase transitions," Journal of the American Ceramic Society, vol. 83, no. 6, pp. 1361-1368, 2000.

[19] J. Yang, S. Mei, and J. M. F. Ferreira, "Hydrothermal synthesis of nanosized titania powders: influence of tetraalkyl ammonium hydroxides on particle characteristics," Journal of the American Ceramic Society, vol. 84, no. 8, pp. 1696-1702, 2001.

[20] J. Yang, S. Mei, and J. M. F. Ferreira, "Hydrothermal synthesis of $\mathrm{TiO}_{2}$ nanopowers from tetraalkylammonium hydroxide peptized sols," Materials Science and Engineering C, vol. 15, no. 1-2, pp. 183-185, 2001.

[21] J. Yang, S. Mei, and J. M. F. Ferreira, "In situ preparation of weakly flocculated aqueous anatase suspensions by a hydrothermal technique," Journal of Colloid and Interface Science, vol. 260, no. 1, pp. 82-88, 2003.

[22] J. Yang, S. Mei, and J. M. F. Ferreira, "Hydrothermal processing of nanocrystalline anatase films from tetraethylammonium hydroxide peptized titania sols," Journal of the European Ceramic Society, vol. 24, no. 2, pp. 335-339, 2004.
[23] J. Yang, S. Mei, and J. M. F. Ferreira, "Hydrothermal synthesis of submicrometer $\alpha$-alumina from seeded tetraethylammonium hydroxide-peptized aluminum hydroxide," Journal of the American Ceramic Society, vol. 86, no. 12, pp. 2055-2058, 2003.

[24] Â. L. Andrade, D. M. Souza, M. C. Pereira, J. D. Fabris, and R. Z. Domingues, " $\mathrm{pH}$ effect on the synthesis of magnetite nanoparticles by the chemical reduction-precipitation method," Quimica Nova, vol. 33, no. 3, pp. 524-527, 2010.

[25] S. H. Hyon, W. I. Cha, and Y. Ikada, "Preparation of transparent poly(vinyl alcohol) hydrogel," Polymer Bulletin, vol. 22, no. 2, pp. 119-122, 1989.

[26] T. Hosono, H. Takahashi, A. Fujita, R. J. Joseyphus, K. Tohji, and B. Jeyadevan, "Synthesis of magnetite nanoparticles for AC magnetic heating," Journal of Magnetism and Magnetic Materials, vol. 321, no. 19, pp. 3019-3023, 2009.

[27] J. C. Bacri, R. Perzynski, V. Cabuil, and R. Massart, "Phase diagram of an ionic magnetic colloid: experimental study of the effect of ionic strength," Journal of Colloid And Interface Science, vol. 132, no. 1, pp. 43-53, 1989.

[28] E. Hasmonay, A. Bee, J. C. Bacri, and R. Perzynski, "pH effect on an ionic ferrofluid: evidence of a thixotropic magnetic phase," Journal of Physical Chemistry B, vol. 103, no. 31, pp. 6421-6428, 1999.

[29] A. L. Patterson, "The scherrer formula for X-ray particle size determination," Physical Review, vol. 56, no. 10, pp. 978-982, 1939.

[30] S. Laurent, S. Dutz, U. O. Häfeli, and M. Mahmoudi, "Magnetic fluid hyperthermia: focus on superparamagnetic iron oxide nanoparticles," Advances in Colloid and Interface Science, vol. 166, no. 1-2, pp. 8-23, 2011.

[31] S. Laurent, D. Forge, M. Port et al., "Magnetic iron oxide nanoparticles: synthesis, stabilization, vectorization, physicochemical characterizations and biological applications," Chemical Reviews, vol. 108, no. 6, pp. 2064-2110, 2008.

[32] R. E. Rosensweig, Ferrohydrodynamics, Cambrige Univerity Press, New York, NY, USA, 1985.

[33] A. Ouasri, A. Rhandour, M. C. Dhamelincourt, P. Dhamelincourt, and A. Mazzah, "Vibrational study of $\left(\mathrm{CH}_{3}\right)_{4} \mathrm{NSbCl}_{6}$ and $\left[\left(\mathrm{CH}_{3}\right)_{4} \mathrm{~N}\right]_{2} \mathrm{SiF}_{6}$," Spectrochimica Acta, vol. 58, no. 12, pp. 2779-2788, 2002.

[34] E. Barrado, F. Prieto, J. Medina, and F. A. López, "Characterisation of solid residues obtained on removal of $\mathrm{Cr}$ from waste water," Journal of Alloys and Compounds, vol. 335, no. 1-2, pp. 203-209, 2002.

[35] J. L. Martín De Vidales, A. López-Delgado, E. Vila, and F. A. López, "Effect of the starting solution on the physico-chemical properties of zinc ferrite synthesized at low temperature," Journal of Alloys and Compounds, vol. 287, no. 1-2, pp. 276283, 1999.

[36] M. Ma, Y. Zhang, W. Yu, H. Y. Shen, H. Q. Zhang, and N. Gu, "Preparation and characterization of magnetite nanoparticles coated by amino silane," Colloids and Surfaces A, vol. 212, no. 2-3, pp. 219-226, 2003.

[37] S. N. Inamdar and S. K. Haram, "Synthesis and characterization of uncapped $\gamma$ - $\mathrm{Fe}_{2} \mathrm{O}_{3}$ nanoparticles prepared by flame pyrolysis of ferrocene in ethanol," Journal of Nanoscience and Nanotechnology, vol. 6, no. 7, pp. 2155-2158, 2006.

[38] M. P. Morales, S. Veintemillas-Verdaguer, M. I. Montero et al., "Surface and internal spin canting in $\gamma-\mathrm{Fe}_{2} \mathrm{O}_{3}$ nanoparticles," Chemistry of Materials, vol. 11, no. 11, pp. 3058-3064, 1999.

[39] Z. Jing, "Preparation and magnetic properties of fibrous gamma iron oxide nanoparticles via a nonaqueous medium," Materials Letters, vol. 60, no. 17-18, pp. 2217-2221, 2006. 
[40] E. Baumgartner, M. A. Blesa, and A. J. G. Maroto, "Kinetics of the dissolution of magnetite in thioglycolic acid solutions," Journal of the Chemical Society, Dalton Transactions, no. 9, pp. 1649-1654, 1982.

[41] S. C. Pang, S. F. Chin, and M. A. Anderson, "Redox equilibria of iron oxides in aqueous-based magnetite dispersions: effect of $\mathrm{pH}$ and redox potential," Journal of Colloid and Interface Science, vol. 311, no. 1, pp. 94-101, 2007.

[42] M. P. Albano and L. B. Garrido, "Dispersion of concentrated aqueous $\mathrm{Si}_{3} \mathrm{~N}_{4}-\mathrm{Y}_{2} \mathrm{O}_{3}-\mathrm{Al}_{2} \mathrm{O}_{3}$ slips with tetramethylammonium hydroxide," Ceramics International, vol. 25, no. 1, pp. 13-18, 1999.

[43] T. Sato, T. Iijima, M. Seki, and N. Inagaki, "Magnetic properties of ultrafine ferrite particles," Journal of Magnetism and Magnetic Materials, vol. 65, no. 2-3, pp. 252-256, 1987.

[44] A. H. Lu, E. L. Salabas, and F. Schüth, "Magnetic nanoparticles: synthesis, protection, functionalization, and application," Angewandte Chemie, vol. 46, no. 8, pp. 1222-1244, 2007. 

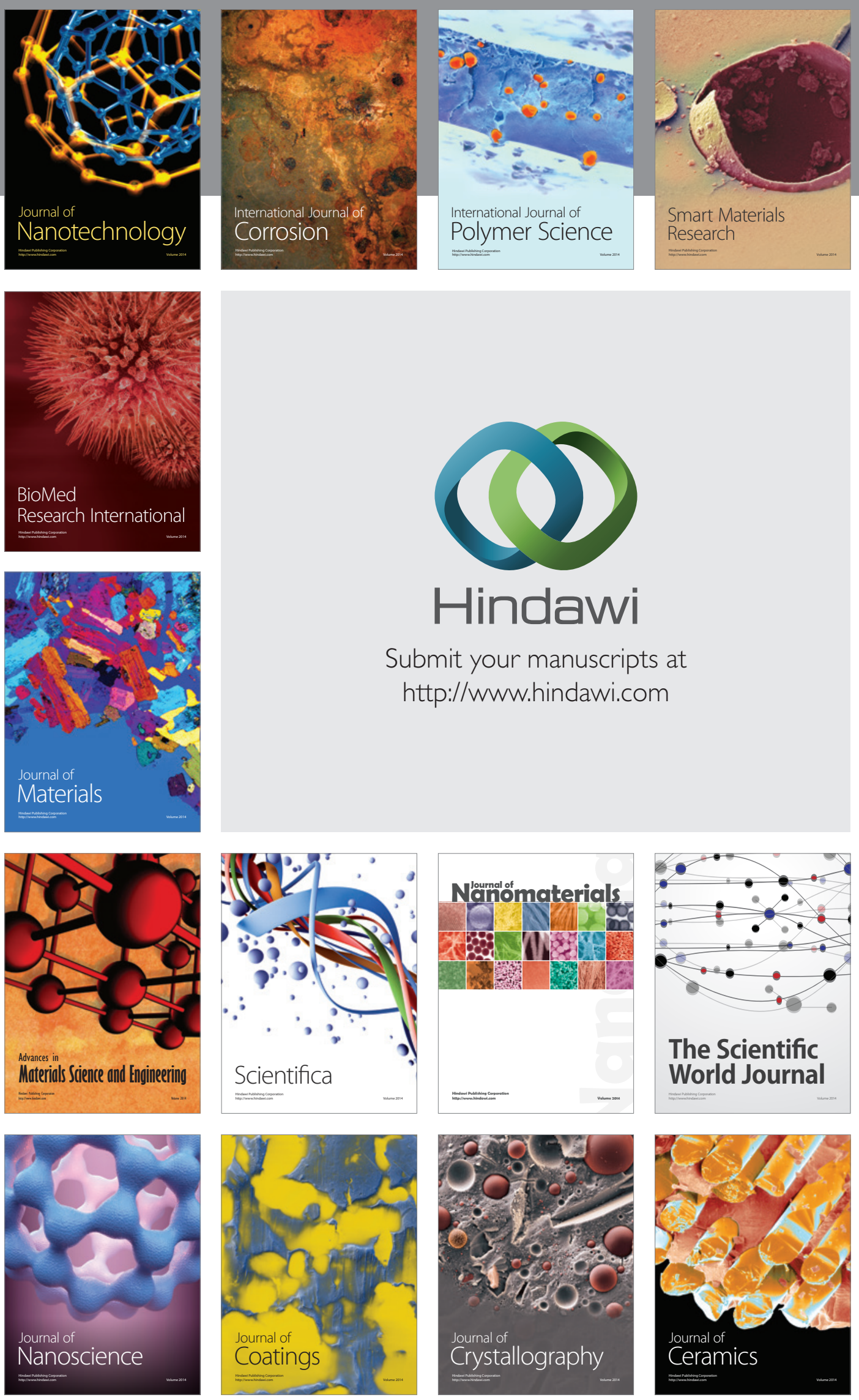

The Scientific World Journal

Submit your manuscripts at

http://www.hindawi.com

\section{World Journal}

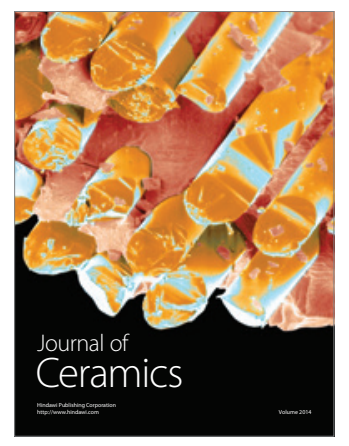

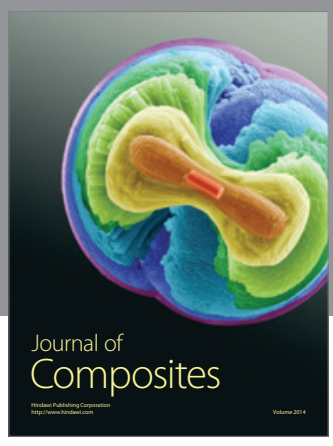
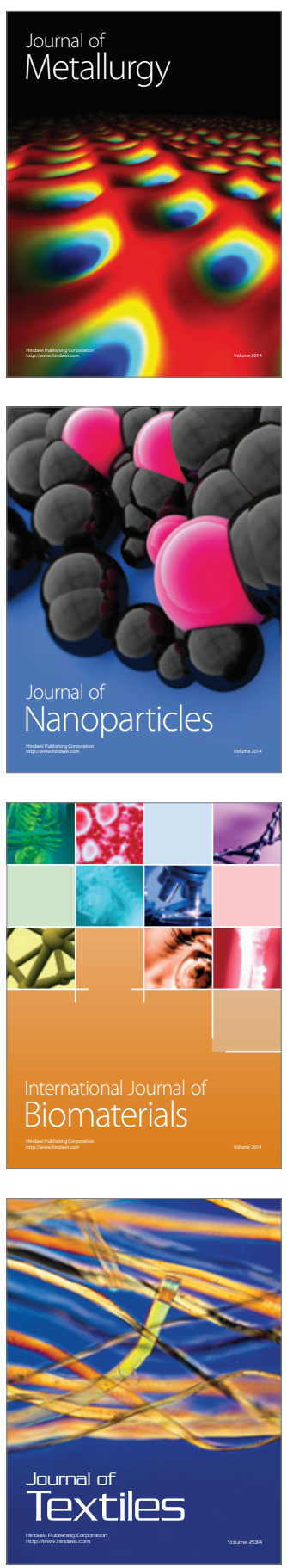